\title{
Inventory rationing and sharing in pre-sell distribution with mobile communication technologies
}

DOI:

10.1016/j.jpe.2007.02.039

\section{Document Version}

Accepted author manuscript

Link to publication record in Manchester Research Explorer

\section{Citation for published version (APA):}

Pishchulov, G., \& Richter, K. (2009). Inventory rationing and sharing in pre-sell distribution with mobile communication technologies. International Journal of Production Economics, 121(2), 584-600.

https://doi.org/10.1016/j.ijpe.2007.02.039

\section{Published in:}

International Journal of Production Economics

\section{Citing this paper}

Please note that where the full-text provided on Manchester Research Explorer is the Author Accepted Manuscript or Proof version this may differ from the final Published version. If citing, it is advised that you check and use the publisher's definitive version.

\section{General rights}

Copyright and moral rights for the publications made accessible in the Research Explorer are retained by the authors and/or other copyright owners and it is a condition of accessing publications that users recognise and abide by the legal requirements associated with these rights.

\section{Takedown policy}

If you believe that this document breaches copyright please refer to the University of Manchester's Takedown Procedures [http://man.ac.uk/04Y6Bo] or contact uml.scholarlycommunications@manchester.ac.uk providing relevant details, so we can investigate your claim.

\section{OPEN ACCESS}




\title{
Inventory rationing and sharing in pre-sell distribution with mobile communication technologies
}

\author{
Grigory Pishchulov*, Knut Richter \\ European University Viadrina, Grosse Scharrnstr. 59, D-15230 Frankfurt (Oder), Germany
}

\begin{abstract}
In pre-sell distribution, the uncertain customer demands are revealed by the company's sales representatives who visit the customers and arrange delivery quantities on the spot, prior to physical execution of deliveries. Given a periodic base-stock of a distributed product, we consider allocation of the product to the customers in two different settings: with and without utilization of mobile communication technologies. There are two performance measures considered: the customer-average fill rate, and the sales profit under service level constraints. The mobile setting is shown to enable a generally better system-wide performance, featuring the capability of inventory pooling. To observe the magnitude of this advantage we determine the optimal allocation policies by means of stochastic dynamic programming. Computational examples for selected configurations and demand distributions are presented.
\end{abstract}

Keywords: Inventory pooling; Inventory rationing; Pre-sell; Mobile communication technologies; Stochastic programming

\section{Introduction}

Distribution of goods is realized in many industries by means of field representatives who travel through assigned territories, discover the customer demand, and execute sales. A predominant role in such distribution practice has been traditionally played by the so called route-sell method, which assumes that a number of vehicles loaded at the depot are sent on tour to meet uncertain customer demands: each vehicle carries the goods on board and distributes (sells) them to the geographically dispersed customers as it follows its route. This distribution mode has been extensively studied in the past decades by the research community in the context of the vehicle routing problem and its stochastic extensions $[4,6,19,25]$.

The presented research deals with an alternative distribution method designated commonly as presell. In pre-sell distribution the sales are arranged with the customers by salespersons in advance, prior to the physical execution of deliveries. These advance salesmen are typically travelling through assigned territories on a regular basis and visit pre-determined customers - like manufacturers, wholesalers and retailers - in order to assess their requirements and "to sell any quantity and every item that might be appropriate" [27], [2,20,38]. They are often assigned to promote products to the customers, check stock on-site, negotiate the sales, and estimate delivery dates [10,38].

Comparing to route-sales, the advance fixing of delivery quantities provides pre-sell with several potential advantages [27,32]: (a) delivery trucks are stocked to accurately match the orders; (b) no stock-outs will occur on the trucks; (c) the trucks carry no excess stock, saving fuel costs; (d) they return empty, therefore no product unloading and handling back at the depot, what saves labor costs and reduces breakage; (e) knowing zero demands in advance means less stops; (f) the routes can be scheduled with more certainty and customers' time windows better met; (g) fewer trucks might be needed. The well known industries that employ pre-sell are e.g. the beverage, soft drink, and consumer goods.

\footnotetext{
*Corresponding author. Tel.: +49335 5534 2605; fax: +49335 5534 2675. E-mail addresses: pishulov@euv-frankfurt-o.de (G. Pishchulov), richter@euv-frankfurt-o.de (K. Richter).
} 
Though the route-sell mode had previously been dominating over pre-sell $[2,20]$, the evidence of the recent years shows pre-sell to win more popularity than before [18], and not least due to novel opportunities enabled by mobile communication technologies. They deliver new attractive capabilities: remote access to the CRM and ERP systems; taking customer orders on-site and transmitting them immediately to the CRM or ERP back-end; tracking roaming employees and assets; communicating customer orders, instructions and data to the right employees in the field [28]. These capabilities result in several new advantages for the enterprise [29,32,36]: (a) a greater speed and accuracy of data collection and transmission; (b) more efficient resource allocation by utilizing real-time data from the field; (c) performance improvements due to a better sourcing of mobile workers with the up-to-date corporate data; (d) a better job assignment.

Convinced by ever growing adoption of mobile solutions [23] and their potential impact on pre-sell distribution, we take in this paper a closer look at the above advantages (b) and (c). For that we consider a company which pre-sells a single good to a number of customers. We show that mobile technologies may act as a means of inventory pooling in pre-sell. Besides that, we let the company use an inventory rationing policy for matching its limited stock against customer demands.

Both pooling and rationing represent important dimensions in inventory management. The concept of inventory pooling has received a remarkable attention in the literature since the publication of the work by Eppen [16], who has shown how consolidation of inventories and aggregation of stochastic demands can reduce the expected holding and penalty costs. Since then many authors have studied pooling strategies in various settings. Alfaro and Corbett [1] give a recent overview for uncapacitated inventory systems. As they point out, much of the literature on such systems assumes normally distributed demands. They make then a detailed analysis of the impact of demand correlation on the value of pooling in an uncapacitated setting with a computational study for some selected non-normal demands. Corbett and Rajaram [12] generalize Eppen's [16] results to non-normal dependent demands. Research on pooling in capacitated systems has been recently advanced by Benjaafar et al. [5]. Typically the analysis of such systems is based on considering Poisson arrivals of unit demands.

Whereas pooling is likely to be a strategic decision, inventory rationing deals with the following, rather operational, problem [13]. If one distinguishes between customer demands of different priority, then one must decide how to allocate the stock to the incoming demands when it runs low: it can sometimes be more rational to stop filling the demands of low priority classes in order to save the stock for meeting possible demands of higher priority - i.e. to ration the inventory. Much of the studies focus namely on determining stationary inventory levels $r_{1}, \ldots, r_{n}$ such that filling the $i$-th class demand stops when the on-hand inventory drops to or below $r_{i}$. Recent reviews of rationing research can be found in [14] and [3].

There also exists research that considers both dimensions of pooling and rationing together. However, this body of research is essentially smaller than that on pooling or rationing only. De Véricourt et al. [13] consider rationing in a production-inventory system with backorders and characterize the optimal policy. They then enable inventory pooling in a system with two demand streams and show that ignoring the stock rationing dimension can lead to wrong managerial decisions. Deshpande et al. [14] run numeric tests to figure out how well their rationing policy does perform in a pooled system with two demand streams compared to a non-pooled system, as well as to a pooled system without rationing. Zhao et al. [40] deal with a decentralized system where independent stocking locations can, besides procuring the items from the manufacturer, also share stock with each other as well as ration it against each other's requests.

Our setting possesses some similarities as well as some distinctions from the existing approaches. Similar to Topkis [37] and Frank et al. [17], we consider an uncapacitated periodic-review base-stock system. Uncertain demands are filled at predetermined customer locations sequentially once a review period. Similar to the assumption made in [17], our policy allows partial filling of uncertain customer demands; the distinction is that we assume all demands are uncertain. The rationing policy that we employ is non-stationary and of a similar nature as in [37]. Utilizing mobile communication technologies, we pool separate stocks and fill customer demands with a rationing policy. We confirm the advantage of pooling in our setting and express it as a function of the base-stock level by means of stochastic dynamic 


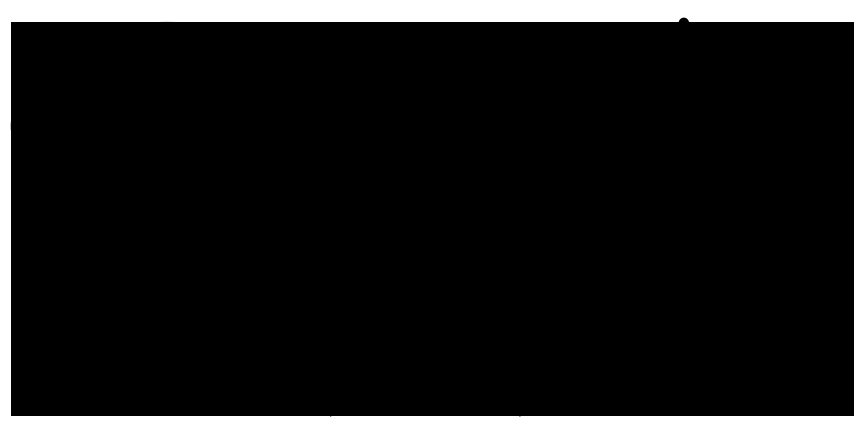

Figure 1: The company serves $K=4$ territories. There are 6 customers in the 1 st territory.

programming. As opposed to most of the studies on pooling or rationing or both, we do not stick to any particular form of demand distribution. The computational method requires the demands to be represented by a finite distribution though, what may assume a discretization of continuously distributed demands.

The rest of the paper is organized as follows. Sections 1.1 and 1.2 introduce a company which pre-sells a single good to a number of customers in two different environments: with and without utilization of mobile communication technologies. In Section 2 we model the company's distribution operations using a stochastic programming framework. Section 3 presents the computational method. In Section 4 we observe the advantage of the mobile setting in a number of numeric examples. In Section 5 we optimize the base-stock policies and make some further comparisons. Section 6 concludes the paper.

\subsection{Decision making without mobile communication technologies}

Let us consider a company that delivers a single good from a central warehouse to $N$ predetermined customers on a regular basis - once a week. The individual weekly demands of the customers are uncertain and represented by random variables $\xi_{i}(i=1, \ldots, N)$ whose joint probability distribution is known. At the beginning of the week, prior to the realisation of any customer demand, the warehouse is being replenished up to the base-stock level $s$.

The company employs $K$ sales representatives (agents) whose jobs consist in visiting the customers, revealing their demands, and arranging delivery quantities. We assume that different representatives are assigned to serve different geographical territories. At the beginning of the week each agent makes a round trip through his territory and visits the assigned customers one by one. A customer's demand becomes first known to the agent upon arrival to this customer. Having learnt the $i$-th customer's demand realisation the agent arranges a delivery quantity $x_{i}$ with him, and drives to the next customer. We assume that each individual demand not necessarily has to be met in full, but is allowed to be met partially in any fraction (e.g., due to a scarce product supply). The uncovered fraction of every individual demand is backlogged and promised for delivery in a later period, upon manufacturing/procurement of the missing merchandise.

Finally, after the delivery quantities for all company's customers have been settled, the physical deliveries are executed.

Even though the customer demands are not known in advance, still each agent should know right before the trip what quantity of the good does he have at his disposal. To secure this the company divides the total stock (physically or virtually) between the agents according to some predefined rule, e.g. in proportion to the expected demands of the territories. Figure 1 sketches an example of such distribution system, showing the separated stock quotas of the territories and the round trips of two representatives. We will call the presented operating mode of the company non-mobile, and say as well the company operates in a non-mobile environment.

Since each agent has a limited resource supply at his command, in general not every individual demand can be fully met by the agent. What should be the decision policy for an agent to use while deciding on a product allocation for the current customer? Should he allocate to the customer as much 
as she demands or should he save more for the customers not yet visited - i.e. ration the inventory? We assume that each representative makes each time such a decision that is intended to maximize the expected overall performance of the company. Let us consider the following two performance measures.

\section{Performance measure 1: customer-average fill rate}

Having found the individual demand realisations in this week to be $\xi_{1}, \ldots, \xi_{N}$, and having met them in amounts $x_{1}, \ldots, x_{N}$, respectively, the company expresses the quality of this week's product allocation $\mathbf{x}=\left(x_{1}, \ldots, x_{N}\right)$ ex-post as

$$
G^{1}(\mathbf{x} \mid \xi)=\frac{x_{1}}{\xi_{1}}+\ldots+\frac{x_{N}}{\xi_{N}}
$$

where any $\xi_{i}=0$ implies $x_{i}=0$ and the corresponding fraction equals 1 by definition. Note that (1), if being multiplied by the scaling factor $1 / N$, can be interpreted as a customer-average fill rate as well as an average customer satisfaction in the given week, and as an average perceived product availability, too. So the company is striving to expose a maximal product availability with a given stock $s$. We further omit the scaling factor for the sake of simplicity.

\section{Performance measure 2: the sales profit}

Here we assume that the company is striving to maximize its sales profit while guaranteeing a certain product availability to every customer, i.e. declaring that each individual demand shall be met at least to a certain fraction $\beta$ of it, $0 \leq \beta \leq 1$. Note that this guaranteed service level acts as a degree up to which inventory rationing is permissible. Since the periodic stock $s$ may turn out to be insufficient for holding the declared service level $\beta$, the company may resort to an urgent (emergency) procurement of the product at a higher cost. Let the ordinary stock bring a profit $\pi_{1}$ per unit delivered to the customer within this week, the extra supply a profit $\pi_{2}$, and the backlogged demand a profit $\pi_{3}$ per unit. We assume $-\infty<\pi_{2}<\pi_{3}<\pi_{1}$ and $\pi_{1}>0$. Then the overall profit expresses in the given week ex-post as

$$
G^{2}(\mathbf{x}, \mathbf{y} \mid \xi)=\sum_{i=1}^{N}\left(\pi_{1} x_{i}+\pi_{2} y_{i}+\pi_{3}\left(\xi_{i}-x_{i}-y_{i}\right)\right)
$$

where $y_{i}$ denotes the quantity procured urgently for delivery to the $i$-th customer, $i=1, \ldots, N$. Note that the assumption on emergency ordering is not restricting: if this option is not available to the company then $\pi_{3}-\pi_{2}$ represents just the unit penalty cost for not holding the declared service level. Also note that we currently do not count the holding costs which the unsold inventory may incur. We incorporate them in our model later in Section 5.

And we raise with respect to each of the performance criteria (1) and (2) the first research question:

Q1: What decision rules should the agents apply in order to deliver a maximal possible performance to the company?

\subsection{Decision making with mobile communication technologies}

Let now all agents make use of mobile computer devices (mobile facilities) able to establish a connection with the company's central computer (the server) at any time and from any customer location. Let each representative be able to upload the information about each allocation to the server right upon its completion. This, in turn, facilitates the company to know at any time the current system state: which customers are still awaiting an allocation and what fraction of the total product supply is still available. Let the agents be also able to retrieve this up-to-date information from the server by means of their mobile devices, at any time and from any customer location.

Then, we can introduce the following mobile operating mode (mobile environment as well). The product stock $s$ is not divided between the agents anymore. Instead, each agent retrieves upon arriving to a customer the current system state along with the log of all demands revealed so far in the system, and 
makes an allocation intended to maximize the overall performance. After making a decision he uploads it immediately to the server. Hence, in the mobile environment the representatives use stock $s$ as a joint resource pool. Let us raise the following questions here:

Q2: What are the agents' optimal decision rules in the mobile operating mode?

Q3: Which operating mode - mobile or non-mobile - is expected to enable a better performance in the sense of criteria (1), (2)? What is the magnitude of this advantage, if any?

\subsection{Strategic behavior of customers and agents}

The assumptions made so far do not preclude the customers and the agents from acting strategically: (a) customers anticipating a scarce product supply can theoretically try to secure better allocations by reporting higher demands than they really have (what is called order inflation); (b) as long as the company delegates a task to the agent there is a certain hazard that he will act opportunistically and do the job due to his own considerations - which may conflict with the company's objective. For example, an agent may try to arrange such favorable allocations for his customers which in effect hurt the overall performance.

The first problem has already received attention in its nature in the supply chain literature. Lee et al. [26] study the causes of the bullwhip effect and show that allocating a limited product supply in proportion to submitted orders causes the buyers to order more than they would if given an unlimited supply, what increases the order variance and should negatively impact the supplier. The authors conjecture, that a different allocation mechanism may eliminate such strategic behavior. Cachon and Lariviere $[8,9]$ study this problem in a capacitated setting in more detail and show that truth-inducing mechanisms exist but fail to maximize the supplier's as well as the buyers' profits. Quite the contrary: they find that a manipulable mechanism would be more beneficial for the supplier since order inflation means a better capacity utilization; in some cases the whole supply chain may even benefit, since more capacity can be built.

The second problem is essentially a principal-agent problem, which has been a subject of study of the agency theory for several decades and can be briefly characterized as follows: the company (principal) is concerned about hiring an employee (agent) on the conditions which would motivate him/her to perform the task in the way the company wants it. Therefore there should some incentives for the agent be created which would align his interests with the company's ones. In this perspective, the relationship between a company and its salesmen has received a remarkable attention in the literature, see [11,39] and references therein. As van Ackere [39] points out, with multiple agents phenomena may arise not observable in the single-agent setting.

We feel that studying the above two questions in the context of the problem given here deserves a research on its own, and we leave these issues initially out of scope of the presented work, restricting our attention to the dimensions of stock sharing and rationing. We therefore assume below that a strategic behavior of the customers and agents is not the case.

\section{Modeling}

\subsection{Non-mobile environment}

Let $n_{1}, \ldots, n_{K}$ denote the customer counts in $K$ geographical territories of the company's distribution system, so that $n_{1}+\ldots+n_{K}=N$. Then, in the non-mobile environment the company divides its stock $s$ into quotas $s_{1}, \ldots, s_{K}$. Each representative visits the customers in his territory one-by-one and executes sequential product allocations out from his quota. Let us denote such non-mobile configuration of the distribution system by $\mathrm{C}\left|n_{1}\right| \ldots\left|n_{K}\right|$. Figure 2(a) illustrates a configuration $\mathrm{C}|2| 2|2|$.

Since the representatives work separately of each other, they can deliver a maximal possible overall performance if and only if each of them maximizes the performance in his territory. So we first turn to decision making of a single representative who has an initial product supply $s_{k}$ and provides service to 


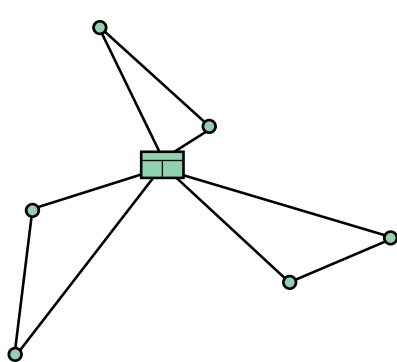

(a) non-mobile

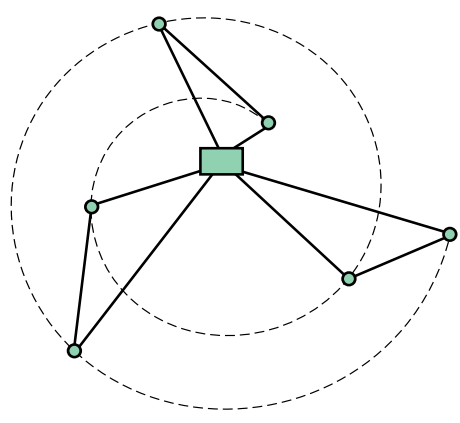

(b) mobile

Figure 2: Transition from non-mobile to mobile operating mode

$n_{k}$ customers. We do this below with respect to each of the performance measures (1) and (2). For the sake of brevity let us use the notation $s, n$ in place of $s_{k}, n_{k}$, respectively.

\subsubsection{Maximization of the customer-average fill rate}

Thus, we consider an agent who services $n$ customers sequentially and has a resource supply $s$ at his disposal. Formally, his decision policy $\mathbf{x}(\xi)=\left(x_{1}\left(\xi_{1}\right), x_{2}\left(\xi_{1}, \xi_{2}\right), \ldots, x_{n}\left(\xi_{1}, \ldots, \xi_{n}\right)\right)$ is a tuple of functions which determine the sequential product allocations of the agent as he visits his customers oneby-one and reveals their demands. Indeed, a product allocation $x_{1}$ has to be made for the 1 st customer upon learning the demand realisation $\xi_{1}$ and without knowing the demand realisations of the rest of the customers. Therefore the allocation $x_{1}$ can depend on $\xi_{1}$ only, and there has to be a rule $x_{1}\left(\xi_{1}\right)$ defined for making an allocation. An allocation $x_{2}$ is made while knowing the demand realisations $\xi_{1}, \xi_{2}$, hence according to some rule $x_{2}\left(\xi_{1}, \xi_{2}\right)$, and so on, up until the $n$-th customer. The agent looks for such a policy $\mathbf{x}(\xi)$ which is expected to maximize his individual performance in the sense of measure (1). Such policy can be found as a solution to the following problem:

$$
\begin{aligned}
E^{1}|n|(s):=\max _{\mathbf{x}(\xi)} & \mathbb{E}_{\xi}\left[\frac{x_{1}\left(\xi_{1}\right)}{\xi_{1}}+\frac{x_{2}\left(\xi_{1}, \xi_{2}\right)}{\xi_{2}}+\ldots+\frac{x_{n}\left(\xi_{1}, \ldots, \xi_{n}\right)}{\xi_{n}}\right] \\
& x_{1}\left(\xi_{1}\right)+x_{2}\left(\xi_{1}, \xi_{2}\right)+\ldots+x_{n}\left(\xi_{1}, \ldots, \xi_{n}\right) \leq s \\
& 0 \leq x_{i}\left(\xi_{1}, \ldots, \xi_{i}\right) \leq \xi_{i}, \quad i=1, \ldots, n
\end{aligned}
$$

So, in view of uncertain customer demands, we measure the quality of a decision policy as the expected performance that it delivers with respect to the probability distribution of the customer demands. The optimal objective value of the problem represents the maximal expected performance $E^{1}|n|(s)$, which is a function of parameter $s$ incorporated into the model. Further in Section 3 we present a method for solving (3) and obtaining $E^{1}|n|(s)$ using stochastic dynamic programming.

\subsubsection{Maximization of the sales profit}

Let us now consider decision making of an agent maximizing the sales profit in his territory, as defined by (2). As before, the agent services $n$ customers and has a resource supply $s$ at his disposal. We represent a decision policy

$$
(\mathbf{x}(\xi), \mathbf{y}(\xi))=\left(x_{1}\left(\xi_{1}\right), y_{1}\left(\xi_{1}\right), x_{2}\left(\xi_{1}, \xi_{2}\right), y_{2}\left(\xi_{1}, \xi_{2}\right), \ldots, x_{n}\left(\xi_{1}, \ldots, \xi_{n}\right), y_{n}\left(\xi_{1}, \ldots, \xi_{n}\right)\right)
$$

as a tuple of functions determining at each decision stage, what quantity $x_{i}$ should be allocated from the existing stock and what quantity $y_{i}$ should be procured additionally. Both must be decided based only on the demand data that has become available to the agent to that moment. The agent looks for such a policy 
$(\mathbf{x}(\xi), \mathbf{y}(\xi))$ which is expected to maximize his individual performance in the sense of measure (2). Such policy can be found as a solution to the following problem:

$$
\begin{aligned}
\max _{\mathbf{x}(\xi), \mathbf{y}(\xi)} & \mathbb{E}_{\xi}\left[\sum_{i=1}^{n}\left(\pi_{1} x_{i}(\cdot)+\pi_{2} y_{i}(\cdot)+\pi_{3}\left(\xi_{i}-x_{i}(\cdot)-y_{i}(\cdot)\right)\right)\right] \\
& x_{1}\left(\xi_{1}\right)+\ldots+x_{n}\left(\xi_{1}, \ldots, \xi_{n}\right) \leq s \\
& \beta \xi_{i} \leq x_{i}\left(\xi_{1}, \ldots, \xi_{i}\right)+y_{i}\left(\xi_{1}, \ldots, \xi_{i}\right) \leq \xi_{i}, \quad i=1, \ldots, n \\
& x_{i}(\cdot), y_{i}(\cdot) \geq 0, \quad i=1, \ldots, n
\end{aligned}
$$

Again, in view of uncertain customer demands, we measure the quality of a decision policy as the expected performance that it delivers, with respect to the probability distribution of the customer demands. Let us perform the following trivial transformation of the objective function in the above problem. Let $g=\frac{\pi_{3}-\pi_{2}}{\pi_{1}-\pi_{3}}$ (note $g>0$ ). Then, omitting the constant term $\mathbb{E}_{\xi}\left[\pi_{3} \xi_{1}+\ldots+\pi_{3} \xi_{n}\right]$ in the objective function and dividing the rest by $\pi_{1}-\pi_{3}$, we reduce the above problem to:

$$
\begin{aligned}
E^{2}|n|(s):=\max _{\mathbf{x}(\xi), \mathbf{y}(\xi)} \mathbb{E}_{\xi}[ & \left.x_{1}\left(\xi_{1}\right)-g y_{1}\left(\xi_{1}\right)+\ldots+x_{n}\left(\xi_{1}, \ldots, \xi_{n}\right)-g y_{n}\left(\xi_{1}, \ldots, \xi_{n}\right)\right] \\
& x_{1}\left(\xi_{1}\right)+\ldots+x_{n}\left(\xi_{1}, \ldots, \xi_{n}\right) \leq s \\
& \beta \xi_{i} \leq x_{i}\left(\xi_{1}, \ldots, \xi_{i}\right)+y_{i}\left(\xi_{1}, \ldots, \xi_{i}\right) \leq \xi_{i}, \quad i=1, \ldots, n \\
& x_{i}(\cdot), y_{i}(\cdot) \geq 0, \quad i=1, \ldots, n
\end{aligned}
$$

Thus, we can operate with the maximal expected sales profit performance in terms of the optimal objective value $E^{2}|n|(s)$ of (4). Again, this is a function of parameter $s$ incorporated into the model. We refer to solving (4) and obtaining $E^{2}|n|(s)$ explicitly later in Section 3.

\subsection{The mobile environment}

Now let the company utilize mobile facilities in its decision making and denote this new (mobile) configuration by $\mathrm{CM}\left|n_{1}\right| \ldots\left|n_{K}\right|$. The company doesn't divide the stock $s$ anymore but rather uses it as a joint resource pool for centralized product allocations, by utilizing mobile facilities. Figure 2(b) illustrates a mobile configuration $\mathrm{CM}|2| 2|2|$.

Let us assume that the customers are being visited in Figure 2 in the time sequence depicted by the dashed line. Let us imagine there was a single imaginary agent able to visit and service the customers in such sequence. Then we must conclude that this "virtual" agent, being subjected to the maximization of the overall performance, would do the same product allocations from the stock $s$ as the real representatives for the same customers in the mobile environment do, since he would have to substitute for a real agent at each customer site while having the same data for making a decision: the log of observed demands and the system state. Thus the following holds for the mobile environment in general: the representatives imitate together the work of a single virtual agent who visits $N$ customers in the same sequence as they are being serviced by the real representatives.

Let us further without loss of generality assume the above servicing sequence to be $1, \ldots, N$. What decision rules should the representatives follow while servicing the customers in their territories in the mobile environment? Obviously, their product allocations are completely defined by an optimal decision policy for the single "virtual" representative which can be found by solving either (3) or (4), depending on performance measure used, with $n=N$. Let the greatest expected overall performance to achieve in $\mathrm{C} \mathcal{M}\left|n_{1}\right| \ldots\left|n_{K}\right|$ be denoted by $E^{\mathrm{P}} \mathcal{M}\left|n_{1}\right| \ldots\left|n_{K}\right|(s)$, where $\mathrm{P}=1,2$ denotes the measure in use. Then, obviously, the following holds:

$$
E^{\mathrm{P}} \mathcal{M}\left|n_{1}\right| \ldots\left|n_{K}\right|(s)=E^{\mathrm{P}}|N|(s), \quad \mathrm{P}=1,2 .
$$




\subsection{Comparing the environments}

Let us turn at first to the the non-mobile environment. Let $E^{\mathrm{P}}\left|n_{1}\right| \ldots\left|n_{K}\right|(s)$ denote the greatest achievable overall performance in the non-mobile configuration $\mathrm{C}\left|n_{1}\right| \ldots\left|n_{K}\right|$, where $\mathrm{P}=1,2$. Since there are $K$ separate servicing sequences, the following holds:

$$
E^{\mathrm{P}}\left|n_{1}\right| \ldots\left|n_{K}\right|(s)=E^{\mathrm{P}}\left|n_{1}\right|\left(s_{1}\right)+\ldots+E^{\mathrm{P}}\left|n_{K}\right|\left(s_{K}\right), \quad \mathrm{P}=1,2 .
$$

Indeed, let us consider at first the case $P=1$. Let the system-wide enumeration of the customers be kept as defined above in Section 2.2. Let $K$ instances of the problem (3) be written down, one for each representative. In each instance $k=1, \ldots, K$ let us replace indices $1, \ldots, n_{k}$ with those ones assigned to the customers by the system-wide enumeration. Let the latter be $\left\{i_{k, 1}, \ldots, i_{k, n_{k}}\right\}=\mathcal{I}_{k}$, respectively. Then, all $K$ problems can be combined into a single aggregate one: we unify the constraints and sum up the objective functions:

$$
\begin{aligned}
\max _{\mathbf{x}(\xi)} & \mathbb{E}_{\xi}\left[\frac{x_{1}(\cdot)}{\xi_{1}}+\frac{x_{2}(\cdot)}{\xi_{2}}+\ldots+\frac{x_{N}(\cdot)}{\xi_{N}}\right] \\
& \sum_{i \in \mathcal{I}_{1}} x_{i}\left(\xi_{j} \mid j \leq i, j \in \mathcal{I}_{1}\right) \leq s_{1} \\
& \ldots \ldots \ldots \ldots \ldots \ldots \ldots \ldots \ldots \ldots \ldots \ldots \\
& \sum_{i \in I_{K}} x_{i}\left(\xi_{j} \mid j \leq i, j \in \mathcal{I}_{K}\right) \leq s_{K} \\
& 0 \leq x_{i}(\cdot) \leq \xi_{i}, \quad i=1, \ldots, N
\end{aligned}
$$

The optimal objective value of (7) is $E^{1}\left|n_{1}\right| \ldots\left|n_{K}\right|(s)$ by definition, what proves (6) to hold for $\mathrm{P}=1$.

Let us now turn to the mobile configuration $\mathrm{CM}\left|n_{1}\right| \ldots\left|n_{K}\right|$. As equation (5) suggests, the maximal achievable expected performance can be found as the optimal objective value $E^{1}|N|(s)$ of the problem (3) with $n=N$. The latter is at the same time a relaxation of problem (7) since it replaces the first $K$ constraints of (7) with just a single one. Therefore, the optimal decision policy is computed in the mobile case over a broader solution set, what in turn means that the optimal objective value in the mobile environment is at least as good as that in the non-mobile case.

The same reasoning as just given can easily be conducted for the case $P=2$, too. This leads us finally to formulating the following proposition. We omit here its rigorous proof.

Proposition 1. $\forall s \geq 0 \quad \forall \mathrm{P}=1,2 \quad: \quad E^{\mathrm{P}}\left|n_{1}\right| \ldots\left|n_{K}\right|(s) \leq E^{\mathrm{P}} \mathcal{M}\left|n_{1}\right| \ldots\left|n_{K}\right|(s)$.

Let us briefly summarize the findings of the current Section.

Firstly, we have shown that questions Q1 and Q2 become resolved for performance measure (1) if we are able to solve problem (3) with the parameters $\left(n_{1}, s_{1}\right), \ldots,\left(n_{K}, s_{K}\right)$ and $(N, s)$, respectively. The same holds for performance measure (2) and problem (4).

Secondly, we have shown with respect to question Q3 the advantage of mobile environment in the sense of both performance measures. We could quantify this advantage for any initial resource supply $s$ if we could compute $E^{\mathrm{P}}\left|n_{1}\right| \ldots\left|n_{K}\right|(s)$ and $E^{\mathrm{P}}|N|(s)$ for both $\mathrm{P}=1,2$, what reduces again to solving problems (3) and (4).

To observe the magnitude of this advantage for all admissible values of $s$, we need to have $E^{\mathrm{P}}\left|n_{1}\right| \ldots\left|n_{K}\right|(s)$ and $E^{\mathrm{P}}|N|(s)$ as functions of $s$. That is, a parametric solution of (3) and (4) comes in question.

\section{Computational method}

In this section we present computational procedures for solving problems (3), (4) and obtaining the functions $E^{\mathrm{P}}|n|(s), \mathrm{P}=1,2$. Let us note first that solutions to both problems are sought in functional spaces. So if random parameters of the models have infinitely many possible realisations (as in the case of continuous demand distributions) then we face an infinite-dimensional optimization problem. This applies 
to stochastic programs in general; the complexity inherent makes them (except for very simple cases) intractable analytically and not amenable to non-linear optimization techniques [21,24,30]. Therefore the usual approach in stochastic programming consists in approximating the underlying probability distribution with a finite discrete one [7, p. 288]. We follow this scheme in elaborating the computational method and make the following basic assumption here: we assume the customer demands to be represented by discrete random variables whose joint distribution has a finite support. This approach provides us with a method which applies equally well to any demand distribution; the trade-off is though that an approximate distribution would mean an approximate solution to the problem. The reader is referred to Dupačová et al. [15] for a survey of methods dealing with discrete representations of underlying random data.

The following two sections present the method for the case of independent customer demands; in Section 3.4 we show how the method can be extended to cover the case of dependent demands.

\subsection{Performance measure 1: customer-average fill rate}

We will use notation $E|n|(s)$ in place of $E^{1}|n|(s)$ in this section for the sake of brevity.

Consider decision making of an agent who has an initial resource supply $s$ and makes the 1st allocation in the sequence of $n$ customers. Thus, $\xi_{1}$ is deterministically known, and there has a decision $x_{1}$ to be made. With $\xi_{1}, x_{1}$ being deterministic we can still utilize (3) for determination of an optimal $x_{1}$. Alternatively, (3) can be rewritten as the following non-linear program (see also [35, pp. 19, 36], [33, pp. 93-94]):

$$
\begin{gathered}
Z_{n}\left(\xi_{1}, s\right):=\quad \max \frac{x_{1}}{\xi_{1}}+E|n-1|\left(s-x_{1}\right) \\
x_{1} \leq s \\
0 \leq x_{1} \leq \xi_{1}
\end{gathered}
$$

Thus, making a decision $x_{1}$, the agent maximizes the satisfaction of the 1st customer plus the expected overall satisfaction of the rest of them with the remaining supply $s-x_{1}$. Obviously,

$$
E|n|(s)=\mathbb{E}_{\xi_{1}}\left[Z_{n}\left(\xi_{1}, s\right)\right]
$$

How can we solve (8) without knowing the embedded function $E|n-1|(s)$ ? Obviously, the latter can be expressed recursively by applying $n:=n-1$ to (9) and (8). Resolving this recursion for all $n:=n-1, n-2, \ldots, 2,1$, we obtain a sequence of nested problems. The most outer problem (8) is then called a multi-stage stochastic program with recourse, namely an $n$-stage one. See more on stochastic programs in $[7,22,34]$.

The nested structure of (8) suggests the idea of a backward recursive computation of $E|n|(s)$ and optimal decision rules $x_{i}\left(\xi_{i}, s\right)$ by stochastic dynamic programming. Below we give a brief outline of such computational procedure; for its detailed presentation see [31, pp. 48-58].

Iteration 1. Start with $n=1$. Define $E|0|(s) \equiv 0$ and consider (8), which is a linear program. For each realization of random demand $\xi_{1}$ express the optimal decision $x_{1}\left(\xi_{1}, s\right)$ and the optimal objective value $Z_{1}\left(\xi_{1}, s\right)$ as functions of $s$. Express $E|1|(s)=\mathbb{E}_{\xi_{1}}\left[Z_{1}\left(\xi_{1}, s\right)\right]$. It proves to be a piecewise linear, non-decreasing, concave function, becoming constant up from a certain value of $s$.

Iteration n. Induction step: assume $E|n-1|(s)$ to be obtained in the previous iteration as piecewise linear, non-decreasing, and concave in $s$. Consider (8). Note $E|n-1|\left(s-x_{1}\right)$ is a piecewise linear, nonincreasing, and concave function in $x_{1}$. Therefore the objective function is piecewise linear and concave with slopes invariant of $s$. For each realization of random demand $\xi_{1}$ express the optimal decision $x_{1}\left(\xi_{1}, s\right)$ and the optimal objective value $Z_{n}\left(\xi_{1}, s\right)$ as functions of $s$. Express $E|n|(s)=\mathbb{E}_{\xi_{1}}\left[Z_{n}\left(\xi_{1}, s\right)\right]$. It proves to be a piecewise linear, non-decreasing, concave function, becoming constant up from a certain value of $s$. 


\subsection{Performance measure 2: the sales profit}

Again, we will use notation $E|n|(s)$ in place of $E^{2}|n|(s)$ in this section for the sake of brevity. We follow here the same scheme as in the previous section.

In order to determine the 1st allocation in the sequence of $n$ customers, we can rewrite (4) as the following non-linear program:

$$
\begin{aligned}
Z_{n}\left(\xi_{1}, s\right):=\quad & \max x_{1}-g y_{1}+E|n-1|\left(s-x_{1}\right) \\
& x_{1} \leq s \\
& \beta \xi_{1} \leq x_{1}+y_{1} \leq \xi_{1} \\
& x_{1}, y_{1} \geq 0
\end{aligned}
$$

So, making a decision $\left(x_{1}, y_{1}\right)$, the agent maximizes the profit earned at the 1st customer plus the profit expected to earn in the next $n-1$ stages with the remaining supply $s-x_{1}$. It holds, as before,

$$
E|n|(s)=\mathbb{E}_{\xi_{1}}\left[Z_{n}\left(\xi_{1}, s\right)\right]
$$

Resolving $E|n|(s)$ for $n:=n-1, n-2, \ldots, 2,1$ recursively using (11) and (10), we obtain a sequence of nested problems embedded into (10) which becomes a multi-stage stochastic program.

We again exploit the nested structure of (10) in a backward recursive computation of $E|n|(s)$ and of optimal decision rules $x_{i}\left(\xi_{i}, s\right), y_{i}\left(\xi_{i}, s\right)$ for each stage of the sequence:

Iteration 1. Start with $n=1$. Define $E|0|(s) \equiv 0$ and consider (10), which is a linear program. For each realization of random demand $\xi_{1}$ express the optimal decision $x_{1}\left(\xi_{1}, s\right), y_{1}\left(\xi_{1}, s\right)$ and the optimal objective value $Z_{1}\left(\xi_{1}, s\right)$ as functions of $s$ explicitly. Express $E|1|(s)=\mathbb{E}_{\xi_{1}}\left[Z_{1}\left(\xi_{1}, s\right)\right]$. It proves to be a piecewise linear, non-decreasing, concave function, becoming constant up from a certain value of $s$.

Iteration n. Induction step: assume $E|n-1|(s)$ to be obtained in the previous iteration as piecewise linear, non-decreasing, and concave in $s$. Consider (10). $E|n-1|\left(s-x_{1}\right)$ is a piecewise linear, nonincreasing, and concave function in $x_{1}$. Therefore the objective function is piecewise linear and concave in both $x_{1}, y_{1}$, and its slopes are invariant of $s$. For each realization of random demand $\xi_{1}$ express the optimal decision $x_{1}\left(\xi_{1}, s\right), y_{1}\left(\xi_{1}, s\right)$ and the optimal objective value $Z_{n}\left(\xi_{1}, s\right)$ as functions of $s$ explicitly. Express $E|n|(s)=\mathbb{E}_{\xi_{1}}\left[Z_{n}\left(\xi_{1}, s\right)\right]$. It proves to be a piecewise linear, non-decreasing, concave function, becoming constant up from a certain value of $s$.

\subsection{Remarks}

Remark 1 . The company can easily differentiate the priority of customer demands in the profit model by setting an individual service level $\beta$ for each customer, as well as by considering customer-specific unit profits $\pi_{1}, \pi_{2}, \pi_{3}$, since the method does not restrict us to all the same values of these parameters. Still, a slight adjustment of the underlying dynamic formulation (10) would be required.

Remark 2. The method proves the optimal allocation policies to be Markovian, since every sequential decision is only state-dependent. Therefore, keeping record of observed demand realisations is not necessary in the case of independent demands.

Remark 3. Since the method is implemented as a backward recursion, an optimal allocation policy for an $i$-th customer in the sequence of visits $1, \ldots, i, \ldots, n$ is determined in the iteration no. $n-i+1$, which treats this customer as standing first in the tail $i, \ldots, n$ of the whole sequence, see Figure 3.

\subsection{Accounting for dependent demands}

So far we have considered the case of independently distributed demands since the model and the solution method are considerably simplified in this case. Still, our approach can be generalized to the case of 


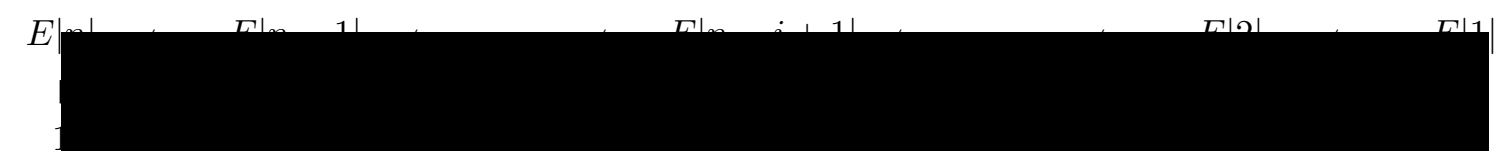

Figure 3: Backward computation of expected performance functions

dependent demands, too. The key issue in making a decision at any stage of the sequence becomes the fact that the probabilities of demand realisations in all subsequent stages depend on the demand realisations observed so far.

Therefore, given a servicing sequence with dependent customer demands, we need to turn the expectations into conditional expectations in the recursions (8)-(9) and (10)-(11). Let $\xi^{i}:=\left(\xi_{1}, \ldots, \xi_{i}\right)$ denote the vector of demands realised up to the $i$-th stage of the sequence. Then the dynamic programming formulations, corresponding to the $i$-th stage decision in a sequence of $n$ customers, take on the following form for performance measures (1) and (2), respectively:

$$
\begin{array}{c|c}
Z_{i}^{n}\left(\xi^{i}, s\right):=\max & \frac{x_{i}}{\xi_{i}}+E|n-i|\left(\xi^{i}, s-x_{i}\right) \\
x_{i} \leq s & Z_{i}^{n}\left(\xi^{i}, s\right):=\max x_{i}-g y_{i}+E|n-i|\left(\xi^{i}, s-x_{i}\right) \\
0 \leq x_{i} \leq \xi_{i} & x_{i} \leq s \\
\beta \xi_{i} \leq x_{i}+y_{i} \leq \xi_{i}
\end{array}
$$

Note that the optimal objective values of the above problems now depend not only on the most recent demand realisation $\xi_{i}$ but on the whole history $\xi^{i}$ of demand observations. Taking the expectation of the optimal objective value with respect to the random demand $\xi_{i}$, we obtain - in each of the two cases - the maximal expected performance up from the $i$-th customer over the rest of the sequence, given the inventory level $s$ and demands $\xi_{1}, \ldots, \xi_{i-1}$ realised in all preceding stages:

$$
E|n-i+1|\left(\xi^{i-1}, s\right)=\mathbb{E}_{\xi_{i}}\left[Z_{i}^{n}\left(\xi^{i-1}, \xi_{i}, s\right) \mid \xi^{i-1}\right]
$$

By means of (13) we can resolve both objective functions in (12) recursively. Using the recursion, we, thus, can organize the backward computation of the maximal expected performance $E|n|(s)$ over the entire sequence. The computations can be aligned on the scenario tree of the underlying random demand process. Figure 4 illustrates such computation for a sequence of $n=3$ customers with dependent Bernoulli demands. The tree nodes, apart from the root one, correspond to the possible demand realisations. The nodes are arranged in levels which we enumerate as $0,1, \ldots, n$ from left to right so that the $i$-th level corresponds to the $i$-th stage of the sequence $(i=1, \ldots, n)$. Thus each path from the root to a leaf node represents one possible scenario of sequential demand realisations. The arc labels represent the probabilities of transiting from node to node.

The backward computation proceeds as follows. At each node in the $i$-th level of the scenario tree $(i=n, \ldots, 1)$ we solve an instance of problem (12) - in just the same way as we solve problems (8) and (10) in iteration $n-i+1$ of the method in Sections 3.1 and 3.2, respectively. At the predecessor nodes we then obtain the maximal expected performance according to (13). Due to the space limitations we omit the presentation of iterations in full detail.

Note that Figure 4 demonstrates a converging structure of computations. Compare this with the serial structure in the case of independent demands illustrated by Figure 3. This shows clearly that the Markovian property of optimal policies — enjoyed in the case of independent demands (see Remark 2) does not hold when demands are dependent. Therefore, keeping record of observed demands is necessary in this case.

Let us now think of a distribution system where customer demands are dependent across territories. Interestingly, in this case the advantage of the mobile setting can be split into two components: the value of stock sharing and the value of information sharing. The nature of the first component is known to us: we have discussed it in Section 2.2 and will yet observe it in Section 4. The nature of the second component can be explained in the following way: in the non-mobile setting the agents have no means to let each 


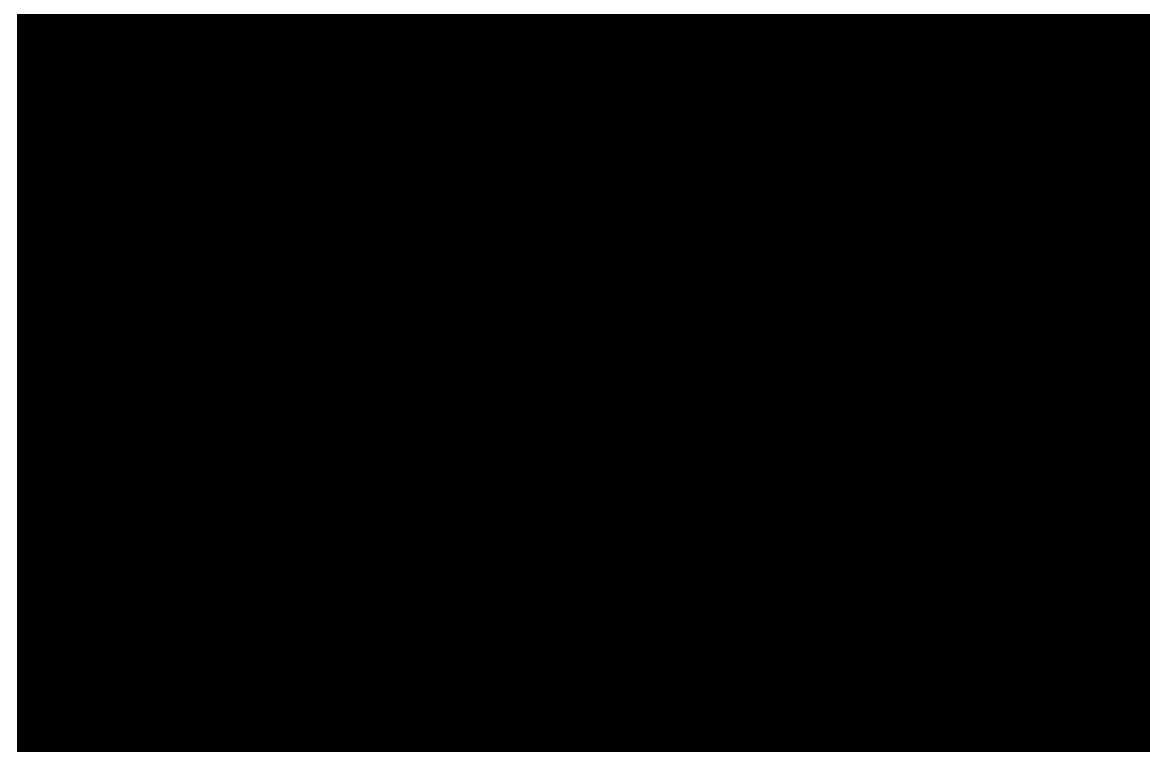

Figure 4: Backward computation of $E|3|(s)$ in the case of dependent Bernoulli demands

other know about the demand realisations in their territories; this information would be helpful though, to make more informed decisions in view of not yet disclosed dependent demands. Such opportunity gets first realised in the mobile environment via the centralised information exchange facilitated by mobile technologies and so creates the value of information sharing. It is interesting for both theoretical and practical reasons to figure out how large each of the two components is. We leave this analysis as an interesting opportunity for a future research and do not consider it here in more detail since it goes beyond the scope of the presented work.

\section{Computational examples}

We consider in this section a number of examples which illustrate the maximal expected overall performance in the non-mobile and mobile environments as functions of the base-stock level $s$. More exactly, we refer to equations (5), (6) and obtain $E^{\mathrm{P}}\left|n_{1}\right| \ldots\left|n_{K}\right|(s)$ and $E^{\mathrm{P}} \mathcal{M}\left|n_{1}\right| \ldots\left|n_{K}\right|(s)$ for both performance measures $P=1,2$ by means of the method presented in Section 3. The difference of the corresponding functions is used then to represent the advantage of the mobile environment. We keep here all customer demands independent and identically distributed.

We prefer to display the maximal expected overall customer satisfaction $E^{1}$ scaled down by $1 / N$ and multiplied by $100 \%$ to make it range from $0-100 \%$ and represent the customer-average expected fill rate (see Section 1.1 on performance measure 1). In other words, we perform the following transformation: $E^{\mathrm{fr}}:=E^{1} \cdot 100 / N(\%)$, and plot the functions $E^{\mathrm{fr}}\left|n_{1}\right| \ldots\left|n_{K}\right|(s)$ and $E^{\mathrm{fr}} \mathcal{M}\left|n_{1}\right| \ldots\left|n_{K}\right|(s)$. The advantage of the mobile setting is then displayed on a separate graph as the absolute difference $E^{\mathrm{fr}} \mathcal{M}-E^{\mathrm{fr}}$.

Maximizing the performance in terms of profit, we stick to the following parameter values: $\beta=0.9$ and $\pi_{1}=6, \pi_{2}=-1, \pi_{3}=1$ (in $\$$ ), what implies $g=0.4$. The maximal expected overall profit is obtained from the function $E^{2}$ by means of the transformation $E^{\mathrm{pr}}:=E^{2} \cdot\left(\pi_{1}-\pi_{3}\right)+\pi_{3} \mathbb{E}\left[\xi_{1}+\ldots+\xi_{N}\right]$ (see derivation of problem (4) in Section 2.1.2). Functions $E^{\mathrm{pr}}$ and $E^{\mathrm{pr}} \mathcal{M}$ are then displayed in the graph. The advantage of the mobile setting is presented as the absolute difference $E^{\mathrm{pr}} \mathcal{M}-E^{\mathrm{pr}}$.

Example 1: $\mathrm{C}|1| 1 \mid$ vs. $\mathrm{CM}|1| 1 \mid$, Bernoulli demands. This is a distribution system with two territories, each containing a single customer whose demand is either 0 or 1 , equally probable. Figure 5 gives the comparison of the customer-average fill rate $E^{\text {fr }}$ between the two configurations in the 1st and the 2nd graphs as described above. The overall profit $E^{\mathrm{pr}}$ is compared in the 3rd and the 4th graphs. Note that in both cases a peak difference is attained if the stock level $s$ equals the mean total demand. If taken relative 
to the non-mobile performance, the peak difference corresponds to a $14 \%$ fill-rate improvement in terms of measure (1), and a 59\% profit increase in terms of measure (2).

Example 2: $C|1| 1|1|$ and $C \mathcal{C M}|1| 1|1|$, Bernoulli demands. See Figure 6. Here the peak difference between the mobile and non-mobile performance functions is attained in an interval around the mean total demand 1.5. The peak difference attained at the stock level $s=1$ corresponds to a ca. $19 \%$ fill-rate improvement in terms of measure (1), and a $107 \%$ profit increase in terms of measure (2), if taken relative to the respective non-mobile performance.

Example 3: 25 customers in 5 territories, discrete uniform demand distribution $\{1,2, \ldots, 10\}$, see Figure 7. Here we present only the difference $E^{\mathrm{fr}} \mathcal{M}-E^{\mathrm{fr}}$ (graph to the left) and $E^{\mathrm{pr}} \mathcal{M}-E^{\mathrm{pr}}$ (graph to the right). Observe that the peak performance increase is attained again when the base-stock is equal to the mean total demand. In this case the relative improvement of the average fill rate is $3.84 \%: 92 \% \approx 4 \%$ whereas the relative profit increase is $\$ 48: \$ 738.8 \approx 6.5 \%$. Functions $E^{1} \mathcal{M}$ and $E^{2} \mathcal{M}$ were found to consist of 251 and 2230 linear segments, respectively. The latter was computed in about 7 seconds on a computer with the AMD Sempron ${ }^{\mathrm{TM}} 1.83 \mathrm{GHz}$ CPU.

Example 4: 65 customers in 5 territories, discrete uniform demand distribution $\{1,2, \ldots, 10\}$, see Figure 8. Again, we present only the difference of the corresponding performance functions. At the peak performance increase, the relative improvement of the average fill rate is $2.32 \%: 96 \% \approx 2.4 \%$ whereas the relative profit increase is $\$ 77: \$ 1992 \approx 3.9 \%$. Functions $E^{1} \mathcal{M}$ and $E^{2} \mathcal{M}$ were found to consist of 651 and 5910 linear segments, respectively. The latter was computed in about 42 seconds on the same computer as in the above example.

The above examples show that the greatest performance increase is realised at the moderate levels of supply/capacity. We can also observe that relative performance improvement grows in the number of territories pooled and declines in the number of customers located in one territory; this evidence, explained by the effect of risk pooling, is consistent with the existing research $[1,16]$.

\section{Determining the optimal base-stock levels}

As we could see in Sections 3 and 4, the expected sales profit $E^{2}|n|(s)$ is increasing in the base-stock level $s$, what may cause an illusion that higher base-stock levels are always more preferable. In this Section we let the unsold inventory incur holding costs at the end of the week, so that an excess stock will have a negative impact on the sales profit in the given period. We then determine the optimal base-stock levels in both mobile and non-mobile environments and compare the resulting overall profits.

So, let the unsold inventory incur holding costs $h$ per unit at the end of the week. Then the expression (2) for the period profit changes to:

$$
\Pi(\mathbf{x}, \mathbf{y} \mid \xi)=\sum_{i=1}^{N}\left(\pi_{1} x_{i}+\pi_{2} y_{i}+\pi_{3}\left(\xi_{i}-x_{i}-y_{i}\right)\right)-h\left(s-x_{1}-\ldots-x_{N}\right) .
$$

The maximal expected sales profit over the sequence of $n$ customers given an initial inventory level $s$ is therefore the optimal objective value of the following problem:

$$
\begin{aligned}
E \Pi|n|(s):=\max _{\mathbf{x}(\xi), \mathbf{y}(\xi)} \mathbb{E}_{\xi}\left[\sum_{i=1}^{n}\left(\pi_{1} x_{i}(\cdot)+\pi_{2} y_{i}(\cdot)+\pi_{3}\left(\xi_{i}-x_{i}(\cdot)-y_{i}(\cdot)\right)\right)-h\left(s-x_{1}(\cdot)-\ldots-x_{n}(\cdot)\right)\right] & \\
& x_{1}\left(\xi_{1}\right)+\ldots+x_{n}\left(\xi_{1}, \ldots, \xi_{n}\right) \leq s \\
& \beta \xi_{i} \leq x_{i}\left(\xi_{1}, \ldots, \xi_{i}\right)+y_{i}\left(\xi_{1}, \ldots, \xi_{i}\right) \leq \xi_{i}, \quad i=1, \ldots, n \\
& x_{i}(\cdot), y_{i}(\cdot) \geq 0, \quad i=1, \ldots, n
\end{aligned}
$$

Let us rearrange the terms of the objective function as follows:

$$
\mathbb{E}_{\xi}\left[\sum_{i=1}^{n}\left(\pi_{1}-\pi_{3}+h\right) x_{i}(\cdot)+\left(\pi_{2}-\pi_{3}\right) y_{i}(\cdot)\right]+\pi_{3} \mathbb{E}_{\xi}\left[\xi_{1}+\ldots+\xi_{n}\right]-h s .
$$




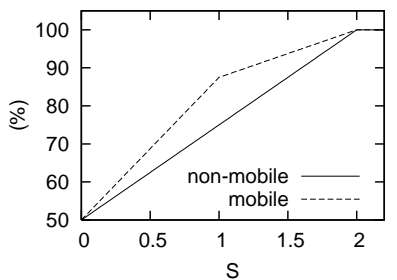

Average fill rate (1)

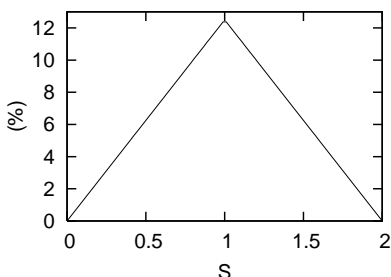

$\rightarrow$ difference

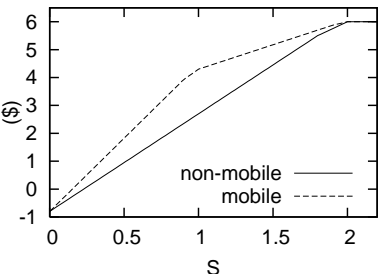

Overall profit (2)

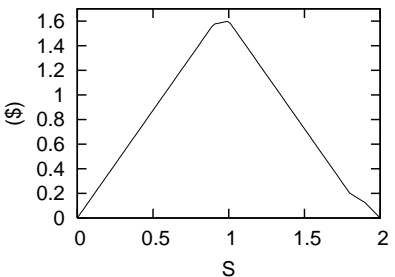

$\rightarrow$ difference

Figure 5: Comparison of performance in $\mathrm{C}|1| 1 \mid$ and $\mathrm{CM}|1| 1 \mid$, Bernoulli demands

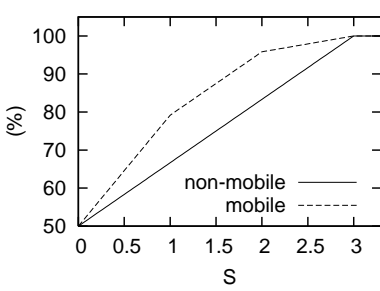

Average fill rate (1)

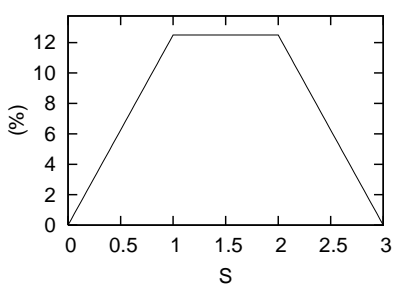

$\rightarrow$ difference

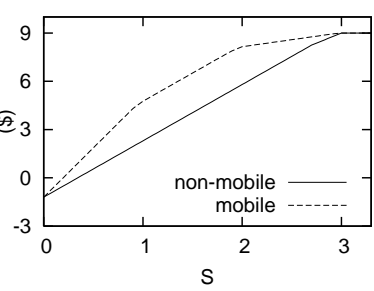

Overall profit (2)

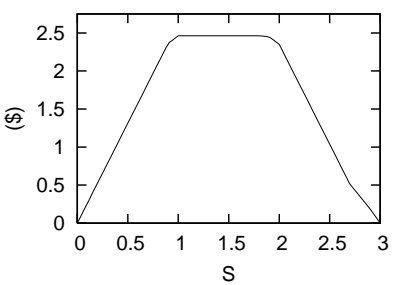

$\rightarrow$ difference

Figure 6: Comparison of performance in $\mathrm{C}|1| 1|1|$ and $\mathrm{CM}|1| 1|1|$, Bernoulli demands

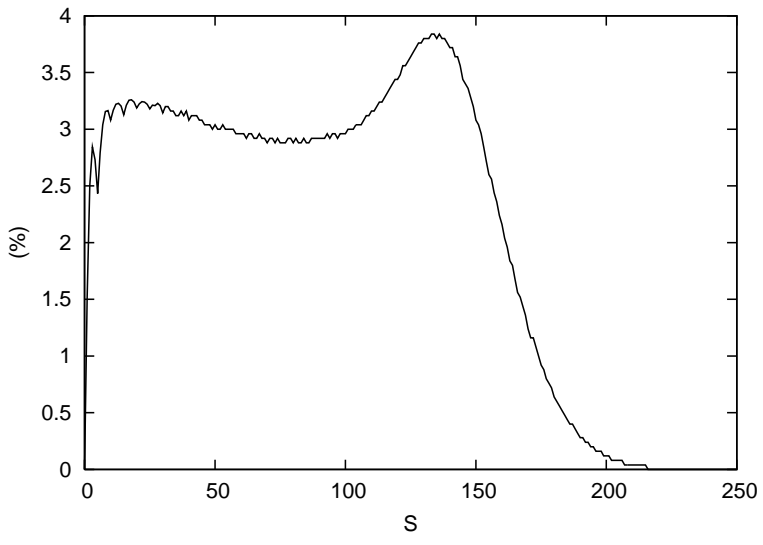

Increase of the average fill rate

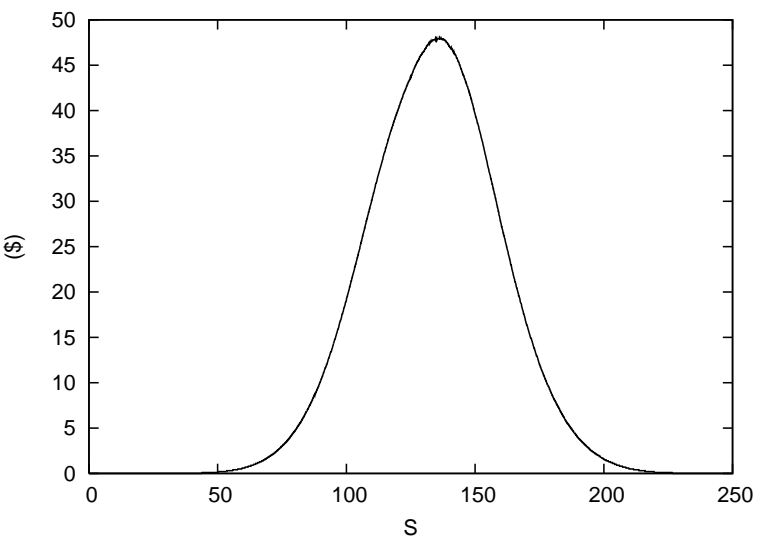

Increase of the overall profit

Figure 7: Advantage of the mobile environment $\mathrm{CM}|5| 5|5| 5|5|(s)$

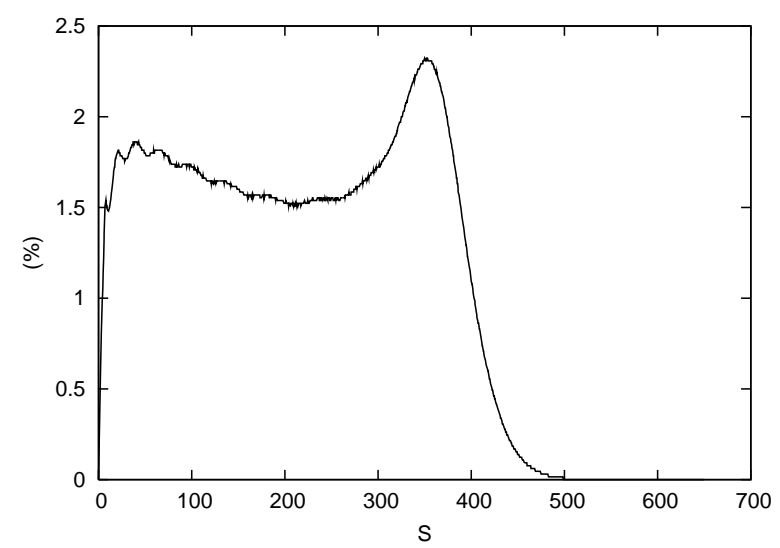

Increase of the average fill rate

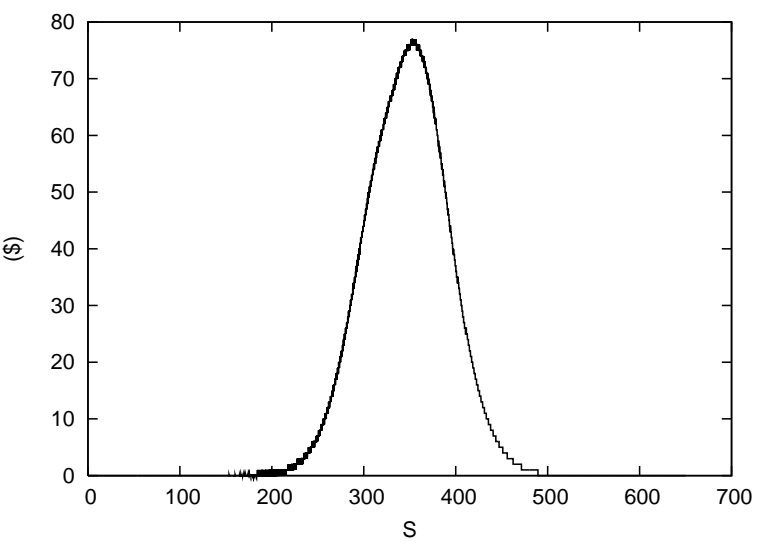

Increase of the overall profit

Figure 8: Advantage of the mobile environment $\mathrm{CM}|11| 12|13| 14|15|(s)$ 
Let us now redefine: $g=\frac{\pi_{3}-\pi_{2}}{\pi_{1}-\pi_{3}+h}$ (note $g>0$ still). Then the objective function of (15) expresses as:

$$
\left(\pi_{1}-\pi_{3}+h\right) \cdot \mathbb{E}_{\xi}\left[\sum_{i=1}^{n} x_{i}(\cdot)-g y_{i}(\cdot)\right]+\pi_{3} \mathbb{E}_{\xi}\left[\xi_{1}+\ldots+\xi_{n}\right]-h s .
$$

As we see, only the first term of (16) depends on the allocation policy in question. Accordingly, the optimal objective value of (15) expresses as:

$$
E \Pi|n|(s)=\left(\pi_{1}-\pi_{3}+h\right) \cdot E^{2}|n|(s)+\pi_{3} \mathbb{E}_{\xi}\left[\xi_{1}+\ldots+\xi_{n}\right]-h s,
$$

where $E^{2}|n|(s)$ is the optimal objective value of problem (4). Note that we are able to compute function $E^{2}|n|(s)$ (and the corresponding optimal allocation policies) by means of the method presented in Section 3.2. Then, from (17), we immediately obtain $E \Pi|n|(s)$ which turns to be the sum of a nondecreasing piecewise linear concave function, a constant, and a decreasing linear function. This proves $E \Pi|n|(s)$ to be piecewise linear and concave. According to the properties of $E^{2}|n|(s)$ derived in Section 3.2, the slopes of its linear segments are non-negative and decrease monotonically down to 0 . As a result, $E \Pi|n|(s)$ switches to a negative slope at some $s^{*}$ due to the presence of the decreasing linear term $-h s$; at this point the non-decreasing behavior of the function changes to a decreasing one. This implies that $s^{*}$ maximizes $E|n|(s)$ on $[0, \infty)$.

Let us now consider the entire distribution system. Due to (5),(6) and (16), the maximal expected system-wide profit expresses in the non-mobile and mobile environments, respectively, as:

$$
\begin{aligned}
E \Pi\left|n_{1}\right| \ldots\left|n_{K}\right|(s) & =E \Pi\left|n_{1}\right|\left(s_{1}\right)+\ldots+E \Pi\left|n_{K}\right|\left(s_{K}\right) \\
E \Pi \mathcal{M}\left|n_{1}\right| \ldots\left|n_{K}\right|(s) & =E \Pi|N|(s)
\end{aligned}
$$

where $s$ denotes the company's base-stock level. With the above equations we are able to optimally choose the base-stock level in each environment. Indeed, in the non-mobile environment the optimal base-stock level is $s_{1}^{*}+\ldots+s_{K}^{*}$ where $s_{k}^{*}$ is the maximizer of $E\left|n_{k}\right|\left(s_{k}\right), k=1, \ldots, K$. In the mobile environment the optimal base-stock level $s^{*}$ is the maximizer of $E|N|(s)$.

Therefore we can compare now how well the company does perform in the mobile and non-mobile environments if the inventory policies are being chosen optimally. We do this below for the distribution systems from Examples 1-4 of the Section 4 . We stick to the same parameter values $\beta=0.9$ and $\pi_{1}=6, \pi_{2}=-1, \pi_{3}=1$ (in $\$$ ) and choose $h=\$ 3$.

Example 1: $C|1| 1 \mid$ and $C \mathcal{M}|1| 1 \mid$, Bernoulli demands. Figure 9(a) depicts functions $E \Pi|1| 1 \mid(s)$ and $E \Pi \mathcal{M}|1| 1 \mid(s)$. The optimal base-stock levels in the non-mobile and mobile environments are found to be 2 and 1 units, delivering the maximal expected sales profits of $\$ 3$ and $\$ 3.55$, respectively. Thus, in the mobile environment the optimal base-stock level is $50 \%$ lower, providing at the same time a $18.33 \%$ average profit increase.

Example 2: $C|1| 1|1|$ and $C \mathcal{M}|1| 1|1|$, Bernoulli demands. Figure 9(b) depicts functions $E \Pi|1| 1|1|(s)$ and $E \Pi \mathcal{M}|1| 1|1|(s)$. The optimal base-stock levels in the non-mobile and mobile environments are found to be 3 and 2 units, delivering the maximal expected sales profits of $\$ 4.5$ and $\$ 6.275$, respectively. Thus, in the mobile environment the optimal base-stock level is about $33 \%$ lower, providing at the same time an almost $40 \%$ average profit increase.

Example 3: 25 customers in 5 territories, discrete uniform demand distribution $\{1,2, \ldots, 10\}$. Figure 9(c) depicts functions $E \Pi|5| 5|5| 5|5|(s)$ and $E \Pi \mathcal{M}|5| 5|5| 5|5|(s)$. The optimal base-stock levels in the nonmobile and mobile environments are found to be 155 and 144 units, delivering the maximal expected sales profits of about $\$ 715.56$ and $\$ 776.68$, respectively. Thus, in the mobile environment the optimal base-stock level is about 7\% lower, providing at the same time an about $8.5 \%$ average profit increase.

Example 4: 65 customers in 5 territories, discrete uniform demand distribution $\{1,2, \ldots, 10\}$. Figure 9 (d) depicts functions $E \Pi|11| 12|13| 14|15|(s)$ and $E \Pi \mathcal{M}|11| 12|13| 14|15|(s)$. The optimal base-stock levels in the non-mobile and mobile environments are found to be 382 and 368 units, delivering the maximal expected sales profits of about $\$ 1970$ and $\$ 2068$, respectively. Thus, in the mobile environment the optimal base-stock level is about $4 \%$ lower, providing at the same time a $5 \%$ average profit increase. 


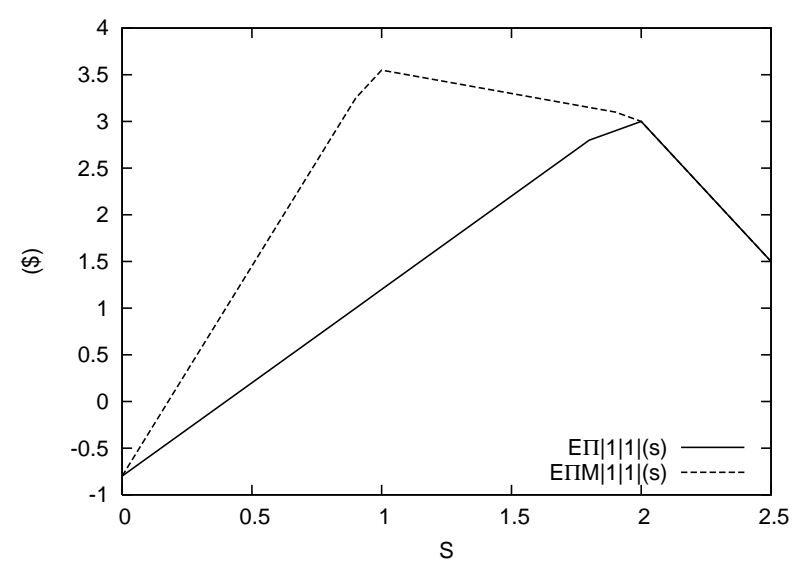

(a) $\mathrm{C}|1| 1 \mid$ vs. $\mathrm{CM}|1| 1 \mid$

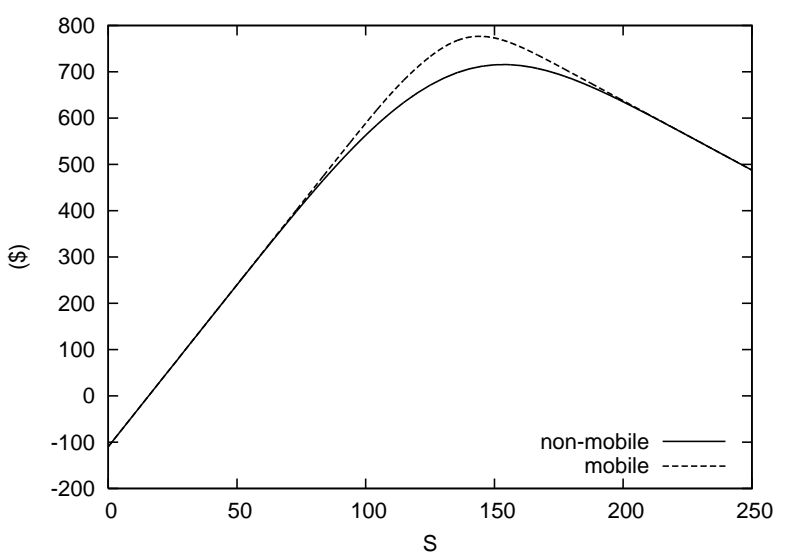

(c) 25 customers in 5 territories

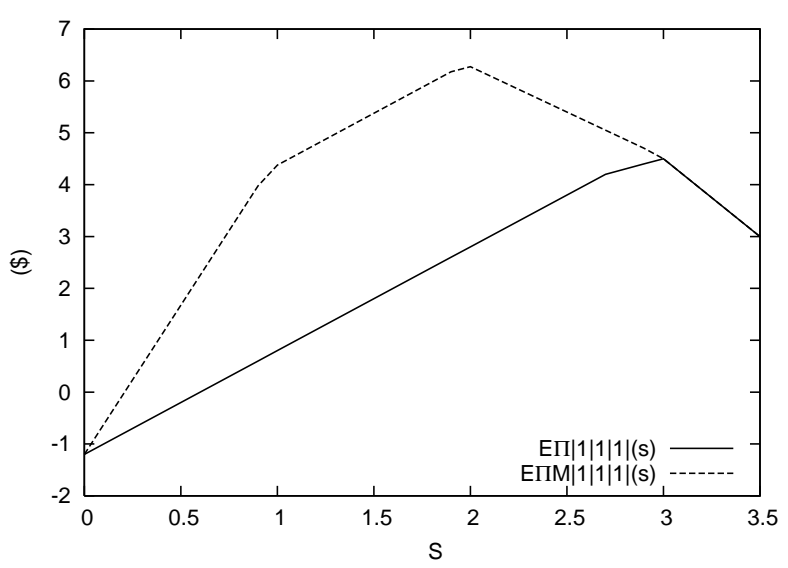

(b) $\mathrm{C}|1| 1|1|$ vs. $\mathrm{C} \mathcal{M}|1| 1|1|$

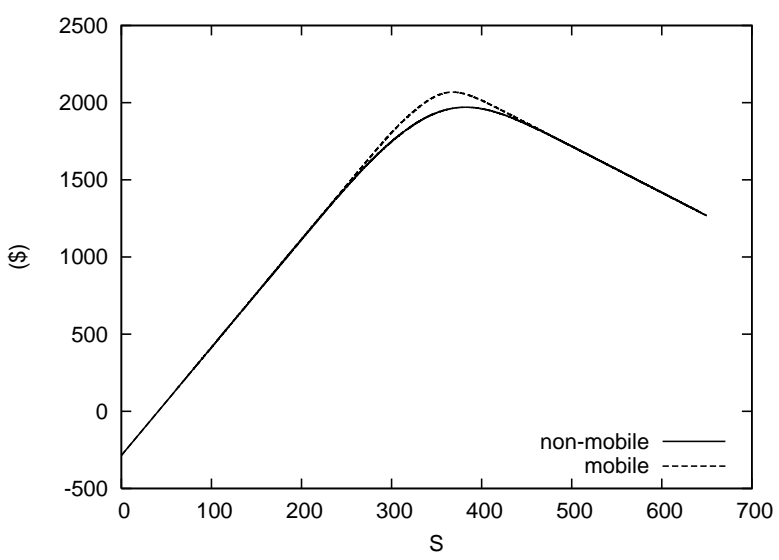

(d) 65 customers in 5 territories

Figure 9: Expected sales profits in the non-mobile and mobile settings with inventory holding costs

\section{Conclusion}

We have considered a periodic-review base-stock system where a stock rationing strategy was used for filling customer demands. The mobile communication technologies were utilized in this system to implement inventory pooling. We have shown how to obtain optimal allocation policies and express the resulting performance for pooled and non-pooled systems. This gave us a possibility to capture the advantage of utilizing mobile facilities as a function of the base-stock level explicitly. We could observe in some numeric examples that utilizing mobile facilities is most beneficial under moderate levels of product supply. We then determined optimality conditions for the base-stock levels and have seen on some examples, how do pooled systems perform compared to their non-pooled counterparts, if their base-stock levels are set up optimally.

The presented model allows to define different customer priorities by choosing customer-individual service levels and sales profit rates without increasing the complexity of the model. Another feature of the presented approach is its capability to efficiently handle every finite probability distribution of customer demands with an option of obtaining approximate solutions in the case of continuous and infinite distributions, too.

There are several directions in which this research can be extended. We emphasize the following ones: studying the impact of demand correlation on the optimal performance; studying how much pooling and rationing each contribute to the performance improvement; incorporating the strategic behavior of customers and agents into the framework. 


\section{Acknowledgements}

The authors are thankful to an anonymous referee for the useful comments and suggestions which helped to improve the paper.

\section{References}

[1] J. A. Alfaro and C. J. Corbett. The value of SKU rationalization in practice (the pooling effect under suboptimal inventory policies and nonnormal demand). Production and Operations Management, 12(1):12-29, 2003.

[2] S. Anily and A. Federgruen. One warehouse multiple retailer systems with vehicle routing costs. Management Science, 36(1):92-114, 1990.

[3] H. Arslan, S. C. Graves, and T. Roemer. A single-product inventory model for multiple demand classes. Working Paper 4542-05, MIT Sloan School of Management, May 2005. http: //hdl . handle .net/1721. 1/17071. Accessed on Mar. 14, 2007.

[4] R. Baldacci, E. Hadjiconstantinou, and A. Mingozzi. An exact algorithm for the capacitated vehicle routing problem based on a two-commodity network flow formulation. Operations Research, 52(5):723-738, 2004.

[5] S. Benjaafar, W. L. Cooper, and J.-S. Kim. On the benefits of pooling in production-inventory systems. Management Science, 51(4):548-565, 2005.

[6] D. J. Bertsimas and D. Simchi-Levi. A new generation of vehicle routing research: robust algorithms, addressing uncertainty. Operations Research, 44(2):286-304, 1996.

[7] J. R. Birge and F. Louveaux. Introduction to Stochastic Programming. Springer, New York, 1997.

[8] G. P. Cachon and M. A. Lariviere. Capacity allocation using past sales: When to turn-and-earn. Management Science, 45(5):685-703, 1999.

[9] G. P. Cachon and M. A. Lariviere. Capacity choice and allocation: Strategic behavior and supply chain performance. Management Science, 45(8):1091-1108, 1999.

[10] California Employment Development Department, USA. California Occupational Guide no. $285,1995$. http://www.calmis.ca.gov/file/occguide/FOODSALE.HTM. Accessed on Mar. 14, 2007.

[11] F. Chen. Salesforce incentives, market information, and production/inventory planning. Management Science, 51(1):60-75, 2005.

[12] C. J. Corbett and K. Rajaram. A generalization of the inventory pooling effect to nonnormal dependent demand. Manufacturing \& Service Operations Management, 8(4):351-358, 2006.

[13] F. de Véricourt, F. Karaesmen, and Y. Dallery. Optimal stock allocation for a capacitated supply system. Management Science, 48(11):1486-1501, 2002.

[14] V. Deshpande, M. A. Cohen, and K. Donohue. A threshold inventory rationing policy for service-differentiated demand classes. Management Science, 49(6):683-703, 2003.

[15] J. Dupačová, G. Consigli, and S. W. Wallace. Scenarios for multistage stochastic programs. Annals of Operations Research, 100(1-4):25-53, 2000.

[16] G. D. Eppen. Effects of centralization on expected costs in a multi-location newsboy problem. Management Science, 25(5):498-501, 1979.

[17] K. C. Frank, R. Q. Zhang, and I. Duenyas. Optimal policies for inventory systems with priority demand classes. Operations Research, 51(6):993-1002, 2003.

[18] E. Goldberg. Drilling down and bubbling up. Beverage World Supplement, 122(5):22-26, May 2003.

[19] B. L. Golden and A. A. Assad, editors. Vehicle Routing: Methods and Studies. North-Holland, Amsterdam, The Netherlands, 1988.

[20] B. L. Golden and E. A. Wasil. Computerized vehicle routing in the soft drink industry. Operations Research, 35(1):6-17, 1987.

[21] P. Kall. On approximations and stability in stochastic programming. In J. Guddat, H. T. Jongen, B. Kummer, and F. Nožička, editors, Parametric Optimization and Related Topics, volume 35 of Mathematical Research, pages 387-407. Akademie-Verlag, Berlin, 1987. 
[22] P. Kall and S. W. Wallace. Stochastic Programming. John Wiley \& Sons, Chichester, 1994.

[23] D. Krebs. Enterprise mobility solutions: Vertical market analyses. Summary of research findings. A white paper, Venture Development Corporation, Dec. 2004. http://www.firstmobiletechnologies.com/ WhitePaper-VerticalMarket.pdf. Accessed on Mar. 14, 2007.

[24] D. Kuhn. Aggregation and discretization in multistage stochastic programming. Stochastic Programming E-Print Series, 2005(18), 2005. http://www .speps .org/.

[25] G. Laporte, F. V. Louveaux, and L. van Hamme. An integer L-shaped algorithm for the capacitated vehicle routing problem with stochastic demands. Operations Research, 50(3):415-423, 2002.

[26] H. L. Lee, V. Padmanabhan, and S. Whang. Information distortion in a supply chain: The bullwhip effect. Management Science, 43(4):546-558, 1997.

[27] S. Makadok. Presell—who needs it? Beverage Industry, 84(12):13, Dec. 1993.

[28] S. Makadok. Software — the hidden advantage. Beverage Industry, 94(3):72, Mar. 2003.

[29] MEI Computer Technology Group Inc. Data collection for merchandisers. http://www.meicpg.com/ Data_Collection_for_Merchandisers.asp. Accessed on Mar. 14, 2007.

[30] G. C. Pflug. Scenario tree generation for multiperiod financial optimization by optimal discretization. Mathematical Programming, 89(2):251-271, 2001.

[31] G. Pichtchoulov and K. Richter. Economic effects of mobile technologies on operations of sales agents. Discussion Paper 226, Europa-Universität Viadrina, Frankfurt (Oder), Germany, Jan. 2005. ISSN 1860 0921. http://www.wiwi.euv-frankfurt-o.de/de/forschung/discussionpaper/226_ Pichtchoulov_Richter.pdf. Accessed on Mar. 14, 2007.

[32] R. Puric and B. Schreib. PepsiAmericas launches next-generation mobile workforce. Wireless Business \& Technology, 2(8), 2002. http://wbt.sys-con.com/read/41117.htm. Accessed on Mar. 14, 2007.

[33] A. Ruszczyński and A. Shapiro. Optimality and Duality in Stochastic Programming, chapter 2, pages 65-139. Volume 10 of Handbooks in Operations Research and Management Science [34], 2003.

[34] A. Ruszczyński and A. Shapiro, editors. Stochastic Programming, volume 10 of Handbooks in Operations Research and Management Science. Elsevier, Amsterdam, 2003.

[35] A. Ruszczyński and A. Shapiro. Stochastic Programming Models, chapter 1, pages 1-64. Volume 10 of Handbooks in Operations Research and Management Science [34], 2003.

[36] TechRepublic, Inc. Enabling the mobile workforce: Issues and trends. A white paper, Feb. 2006. http:// whitepapers.techrepublic.com.com/whitepaper.aspx?\&docid=165945. Accessed on Mar. 14, 2007.

[37] D. M. Topkis. Optimal ordering and rationing policies in a nonstationary dynamic inventory model with $n$ demand classes. Management Science, 15(3):160-176, 1968.

[38] U.S. Bureau of Labor Statistics, USA. Occupational Outlook Handbook, 2006-07 edition. http://www. bls.gov/oco/ocos119.htm. Accessed on Mar. 14, 2007.

[39] A. van Ackere. The principal/agent paradigm: Its relevance to various functional fields. European Journal of Operational Research, 70(1):83-103, 1993.

[40] H. Zhao, V. Deshpande, and J. K. Ryan. Inventory sharing and rationing in decentralized dealer networks. Management Science, 51(4):531-547, 2005. 